\title{
Pulsed Field Magnetization of Single Grain, Bulk YBCO Processed from Graded Precursor Powders
}

\author{
Mark D. Ainslie, Member, IEEE, Hidehiko Mochizuki, Hiroyuki Fujishiro, Wei Zhai, Devendra K. Namburi, \\ Yun-Hua Shi, Jin Zou, Anthony R. Dennis, and David A. Cardwell
}

\begin{abstract}
Large, single-grain bulk high-temperature superconducting materials can trap high magnetic fields in comparison with conventional permanent magnets, making them ideal candidates to develop more compact and efficient devices, such as actuators, magnetic levitation systems, flywheel energy storage systems and electric machines. However, macrosegregation of Y-211 inclusions in melt-processed Y-Ba-Cu-O (YBCO) limits the macroscopic critical current density of such bulk superconductors, and hence, the potential trapped field. A new fabrication technique using graded precursor powders has recently been developed by our research group, which results in a more uniform distribution of Y-211 particles, in order to further improve the superconducting properties and trapped field capability of such materials.

In this paper, experimental results on the pulsed field magnetization (PFM) of a graded, single grain YBCO bulk sample are presented. Pulsed fields of magnitude up to $5.5 \mathrm{~T}$ were applied to the sample at temperatures of $40 \mathrm{~K}$ and $65 \mathrm{~K}$. The trapped field profiles indicate that magnetic flux enters the sample in a more uniform manner than standard YBCO samples, resulting in a higher overall trapped field and indicating the success of this processing technique to produce a more homogeneous sample.
\end{abstract}

Index Terms-Trapped field magnets, bulk superconductors, graded YBCO, pulsed field magnetization, magnetic flux penetration

\section{INTRODUCTION}

T ARGE, SINGLE-GRAIN bulk high-temperature

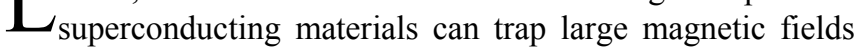
over $17 \mathrm{~T}$ at temperatures below $30 \mathrm{~K}$ [1], [2] and up to $3 \mathrm{~T}$ at the technologically important temperature of $77 \mathrm{~K}$ [3]. This makes them attractive as permanent magnet equivalents (as so-called trapped field magnets) in a number of practical applications, such as rotating machines [4]-[7] and magnetic separation [8]. However, in standard melt-processed Y-Ba-Cu$\mathrm{O}$ (YBCO) samples, macro-segregation of Y-211 inclusions can limit the macroscopic critical current density, $J_{\mathrm{c}}$, and can

M. D. Ainslie would like to acknowledge financial support from a Royal Academy of Engineering Research Fellowship. This work was also supported by a Royal Society International Exchanges Scheme Grant, IE131084. J. Zou would like to acknowledge financial support from Churchill College, the China Scholarship Council and the Cambridge Commonwealth, European and International Trust.

M. D. Ainslie, W. Zhai, D. K. Namburi, Y-H. Shi, J. Zou, A. R. Dennis and D. A. Cardwell are with the Bulk Superconductivity Group, Department of Engineering, University of Cambridge, Cambridge CB2 1PZ, UK (e-mail: mark.ainslie(eng.cam.ac.uk).

H. Mochizuki and H. Fujishiro are with the Department of Materials Science and Engineering, Faculty of Engineering, Iwate University, Morioka 020-8551, Japan (e-mail: fujishiro@iwate-u.ac.jp). also result in a non-uniform $J_{\mathrm{c}}$ distribution throughout the bulk [9]. In order to improve the superconducting material properties of such bulks, our group has been developing a fabrication technique using graded precursor powders in order to achieve a more uniform distribution of Y-211 particles throughout the sample to improve the uniformity of $J_{\mathrm{c}}[10]$. In this paper, we examine the trapped field performance of a graded YBCO sample and compare the results to those of standard YBCO samples, using field cooling (FC) magnetization and pulsed field magnetization (PFM) techniques.

\section{SAMPLE INFORMATION}

For this study, a graded, single grain YBCO sample was fabricated using a buffer-aided, top-seeded melt-growth (TSMG) process [11]-[13]. Two layers of precursor powders were arranged in U-shaped stacks, as shown in Fig. 1, with varying $\mathrm{CeO}_{2}$ content in the top and bottom regions. $\mathrm{CeO}_{2}$ powders are usually added in the TSMG process to reduce the size of Y-211 inclusions and $\mathrm{CeO}_{2}$ particles can react with $\mathrm{Y}$ 211 particles prior to melting to produce $\mathrm{BaCeO}_{3}$ particles that can act as effective pinning centres, both of which act to increase $J_{\mathrm{c}}$ [11]. Precursor powders containing $70 \mathrm{wt} \% \mathrm{Y}-123$ $+30 \mathrm{wt} \% \mathrm{Y}-211$ were used for both stacks, with $2 \mathrm{wt} \% \mathrm{CeO}_{2}$ added in the top region (close to the seed/buffer) and $1 \mathrm{wt} \%$ $\mathrm{CeO}_{2}$ in the bottom region [11]. A buffer pellet is used improve the success rate of the melt-growth process and to aid the growth of the Y-123 phase for improved superconducting properties [12], [13]. The as-grown, pre-polished sample and the sample after polishing and mounting for magnetization experiments are shown in Fig. 2. The final sample diameter is $31.2 \mathrm{~mm}$ and its thickness is $17.7 \mathrm{~mm}$.

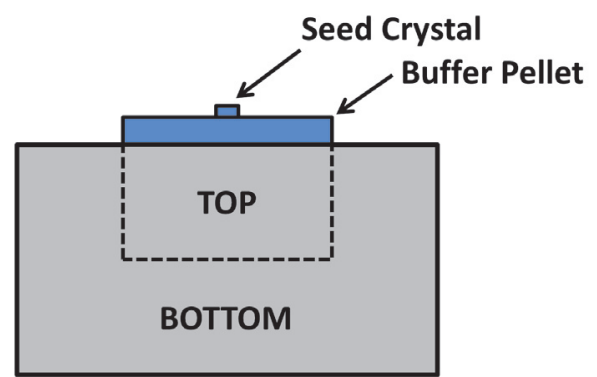

Fig. 1. A graded, bulk YBCO sample fabricated by varying the weight of $\mathrm{CeO}_{2}$ in the precursor powders (top $=2 \mathrm{wt} \%$, bottom $=1 \mathrm{wt} \%$ ) arranged in a nested, U-shaped cylindrical stack. Precursor powders containing $70 \mathrm{wt} \%$ $\mathrm{Y}-123+30 \mathrm{wt} \% \mathrm{Y}-211$ were used for both stacks. A buffer pellet is used to improve processing reliability and the sample's superconducting properties. 

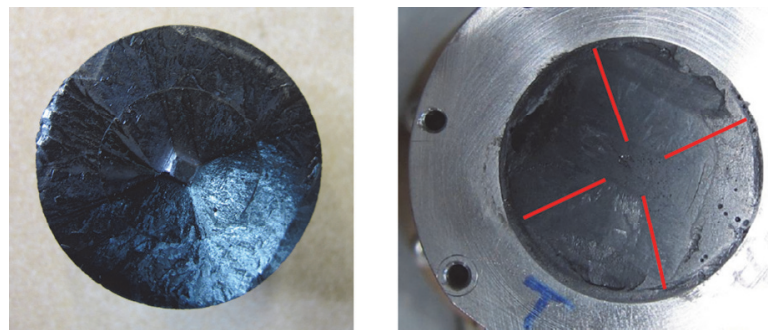

Fig. 2. Photograph of the top surface of the as-grown, pre-polished graded YBCO bulk sample (left) and the sample after polishing and mounting in the stainless steel ring for the pulsed field magnetization experiments (right).

\section{Field Cooling (FC) Magnetization}

The FC magnetization technique gives the best indication of the trapped field capability of a bulk superconductor sample and is useful for comparing the maximum potential of different samples [14], [15]. Fig. 3 shows the FC results for the graded YBCO sample, measured by a Hall sensor placed at the centre of the top surface of the sample, as the temperature is increased from 40 to $100 \mathrm{~K}$ after application of a $7 \mathrm{~T}$ magnetic field. This graded YBCO sample achieved trapped field values of $B_{\mathrm{t}}=5.95 \mathrm{~T}$ at $40 \mathrm{~K}, B_{\mathrm{t}}=2.38 \mathrm{~T}$ at $65 \mathrm{~K}$, and $B_{\mathrm{t}}$ $=0.92 \mathrm{~T}$ at $77 \mathrm{~K}$. For comparison, the results for two standard YBCO samples are also shown: one is a new sample made for this study (diameter $26 \mathrm{~mm}$, thickness $11.6 \mathrm{~mm}$ ) and the other (diameter $32 \mathrm{~mm}$, thickness $15 \mathrm{~mm}$ ) was presented in [16]. Both standard samples were fabricated by the TSMG process, similar to that described in previous research [17].

In order to make a fair and adequate comparison between all of the samples, which are of different dimensions, Fig. 4 presents the FC magnetization results normalized using a correction factor, as described in [14]. Using the Bean model/Biot-Savart law, the original, measured trapped field is divided by the factor $k \mu_{0} R$ to consider the average, in-field critical current density for each sample, averaged over the sample volume. Here $k$ is a geometric constant to account for different sample radii and thicknesses and $R$ is the radius of the sample.

From Figs. 3 and 4, it is clear that large, single grain bulk YBCO can be fabricated using this graded technique with trapped field capabilities equal to or better than standard samples.

\section{Pulsed Field Magnetization (PFM)}

FC and zero field cooling (ZFC) magnetization techniques invariably require large magnetizing coils, which is impractical for most applications of bulk superconductors. The PFM technique, where a large magnetic field is applied via a pulse on the order of milliseconds, currently shows the most promise as a compact, mobile and relatively inexpensive magnetization technique for bulks acting as trapped field magnets (TFMs) in practical applications [18].

An overview of the pulsed field magnetization experimental setup is described in [16]. The magnetizing coil can provide pulsed external fields up to $B_{\mathrm{ex}}=6.4 \mathrm{~T}$, applied parallel to the $\mathrm{c}$-axis of the sample, with a rise time of $t_{\mathrm{r}}=12 \mathrm{~ms}$ and duration of approximately $t_{\mathrm{d}}=120 \mathrm{~ms}$. The trapped field close to the centre of the samples was measured dynamically during the application of each pulsed field using an axial-type Hall sensor located on the top surface of the samples, and two-dimensional (2D) trapped field distributions were measured inside the vacuum chamber using an $x-y$ stage controller and the same Hall sensor positioned approximately $1 \mathrm{~mm}$ above the top surface.

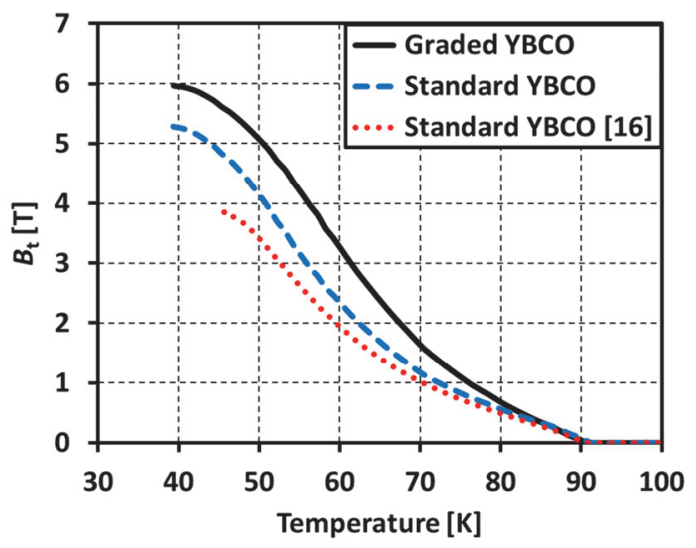

Fig. 3. Field cooling (FC) magnetization results for the graded YBCO sample as the temperature is increased from 40 to $100 \mathrm{~K}$ after application of a $7 \mathrm{~T}$ magnetic field. For comparison, the results for two standard YBCO samples are also shown: one is a new sample made for this study and the other was presented in [16].

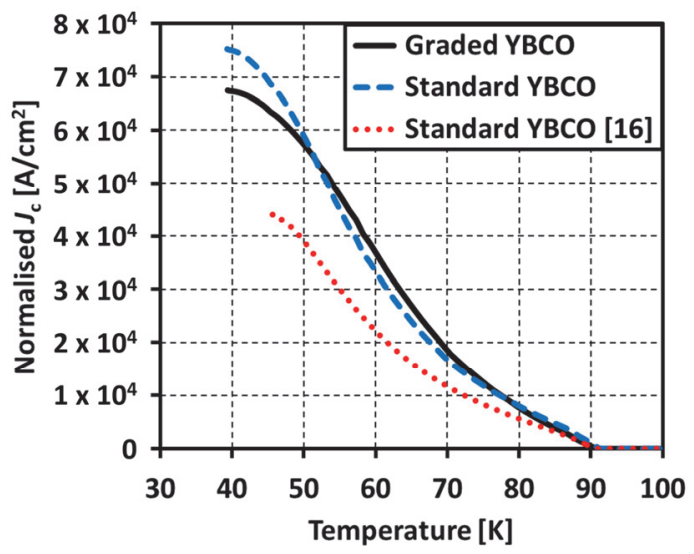

Fig. 4. Field cooling (FC) magnetization results from Fig. 1, normalized using the Bean model/Biot-Savart law as described in [14], to account for different sample geometries.

\section{A. Trapped Field Performance Using PFM}

Figs. 5 and 6 compare the PFM trapped field results for the graded and standard YBCO samples at $65 \mathrm{~K}$ and $40 \mathrm{~K}$, respectively, measured close to the centre of the top surface of the samples. It is clear from these results that the graded sample traps a larger magnetic field than the standard samples, which correlates well with the FC magnetization results in Fig. 3. Furthermore, the full activation field, defined as the external field required to fully magnetize the sample, is lower at both temperatures for the graded sample, suggesting the flux penetrates into this sample in a more uniform manner than for the standard samples. The steep slope of the line at $65 \mathrm{~K}$ also suggests a more uniform penetration of magnetic flux as the trapped field increases suddenly around the full activation 
field. In order to provide further evidence for these hypotheses, we examine the uniformity of the flux penetration in the next section, using the 2D trapped field distributions.

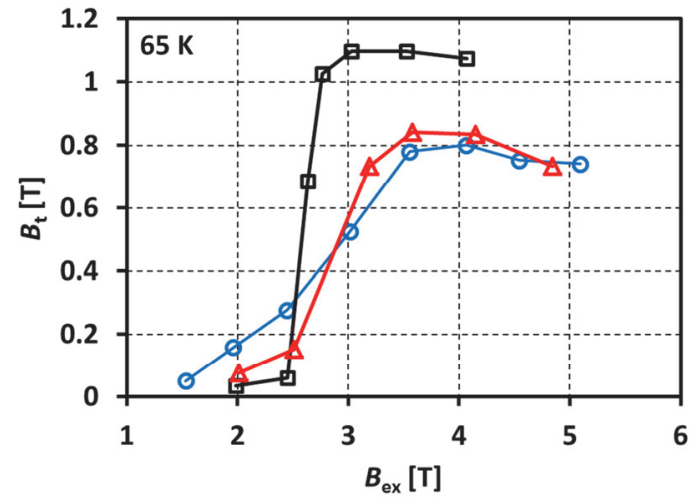

$\bullet$ Standard YBCO $廿$ Graded YBCO $\triangle$ Standard YBCO [16]

Fig. 5. Trapped fields for the three samples at an operating temperature of $65 \mathrm{~K}$, measured at the centre of the top bulk surface $(z=0 \mathrm{~mm})$.



๑ Standard YBCO $廿$ Graded YBCO $\triangle$ Standard YBCO [16]

Fig. 6. Trapped fields for the three samples at an operating temperature of $40 \mathrm{~K}$, measured at the centre of the top bulk surface $(z=0 \mathrm{~mm})$.

\section{B. Two-Dimensional Trapped Field Profiles}

Figs. 7 and 8 show the 2D trapped field profiles for the graded YBCO sample after PFM at $65 \mathrm{~K}$ for an applied pulsed magnetic field lower than the full activation field $\left(B_{\mathrm{ex}}=2.45\right.$ $\mathrm{T})$ and after fully magnetizing the sample $\left(B_{\mathrm{ex}}=3.03 \mathrm{~T}\right)$, respectively. For comparison, Figs. 9 and 10 show the 2D trapped field profiles for the standard YBCO sample for similar applied fields $\left(B_{\mathrm{ex}}=2.45 \mathrm{~T}\right.$, below the full activation field, in Fig. 9 and $B_{\mathrm{ex}}=3.01 \mathrm{~T}$, close to the full activation field, in Fig. 10). It is clear from these observations that the flux penetration into the graded sample is more uniform than for the standard sample, which behaves similarly to the standard sample presented in [16]. This suggests a more homogeneous $J_{\text {c }}$ distribution has been achieved in the graded sample, which is extremely important in order to achieve enhanced, homogeneous trapped fields from bulk superconductors [9]. An inhomogeneous $J_{\mathrm{c}}$ distribution affects dynamics of the flux entering the sample and can reduce the overall trapped field because of localized heating within the sample and preferential movement and pinning of flux lines in regions of low and high $J_{\mathrm{c}}$, respectively [16].

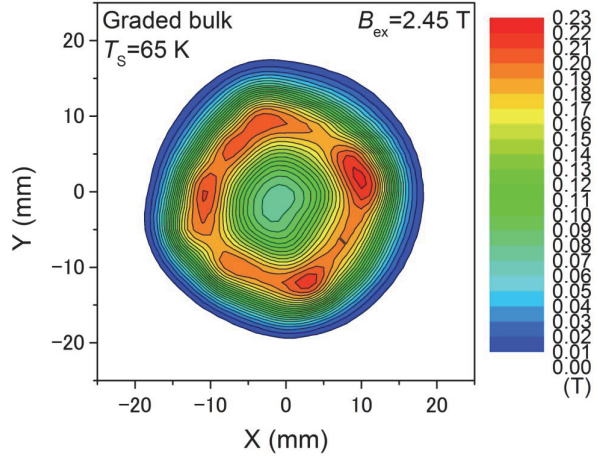

Fig. 7. 2D trapped field profile for the graded YBCO sample after PFM at $65 \mathrm{~K}$ for an applied pulsed magnetic field, $B_{\mathrm{ex}}=2.45 \mathrm{~T}$, measured at $1 \mathrm{~mm}$ above the top surface.

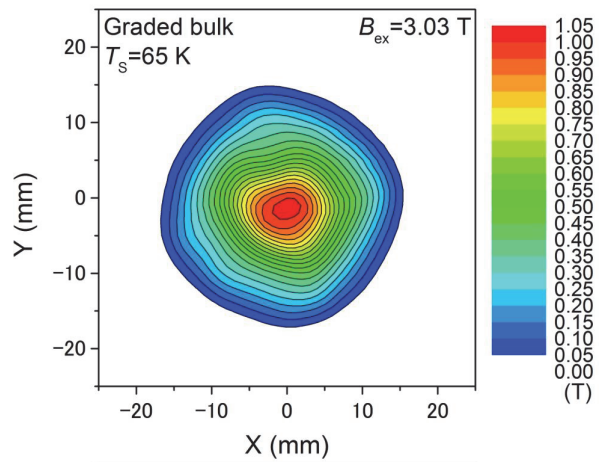

Fig. 8. 2D trapped field profile for the graded YBCO sample after PFM at $65 \mathrm{~K}$ for an applied pulsed magnetic field, $B_{\text {ex }}=3.03 \mathrm{~T}$, measured $1 \mathrm{~mm}$ above the top surface.

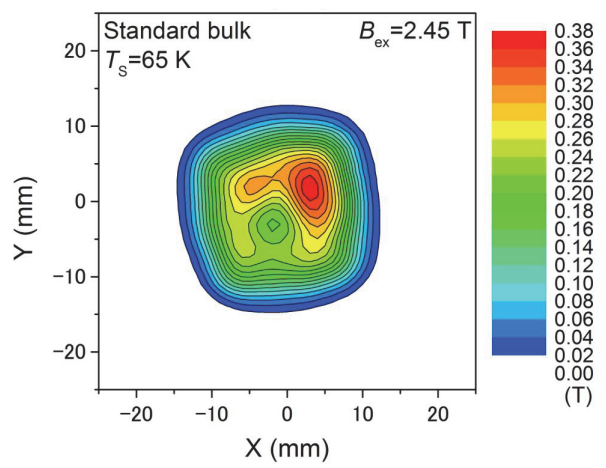

Fig. 9. 2D trapped field profile for the new standard YBCO sample after PFM at $65 \mathrm{~K}$ for an applied pulsed magnetic field, $B_{\mathrm{ex}}=2.45 \mathrm{~T}$, measured $1 \mathrm{~mm}$ above the top surface.

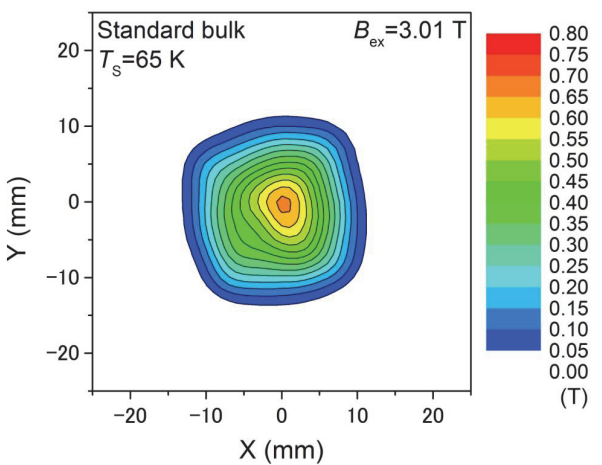

Fig. 10. 2D trapped field profile for the new standard YBCO sample after PFM at $65 \mathrm{~K}$ for an applied pulsed magnetic field, $B_{\mathrm{ex}}=3.01 \mathrm{~T}$, measured 1 $\mathrm{mm}$ above the top surface. 
Figs. 11 and 12 show the 2D trapped field profiles for the graded YBCO sample after PFM at $40 \mathrm{~K}$ for an applied pulsed magnetic field lower than the full activation field $\left(B_{\mathrm{ex}}=3.25\right.$ $\mathrm{T})$ and after fully magnetizing the sample $\left(B_{\mathrm{ex}}=4.09 \mathrm{~T}\right)$, respectively. The lower operating temperature results in stronger flux pinning in the sample, and hence has a larger full activation field. However, the flux penetration shows a similar uniformity to that at $65 \mathrm{~K}$, which is particularly noticeable when compared directly with results for the standard sample (for example, see [16]).

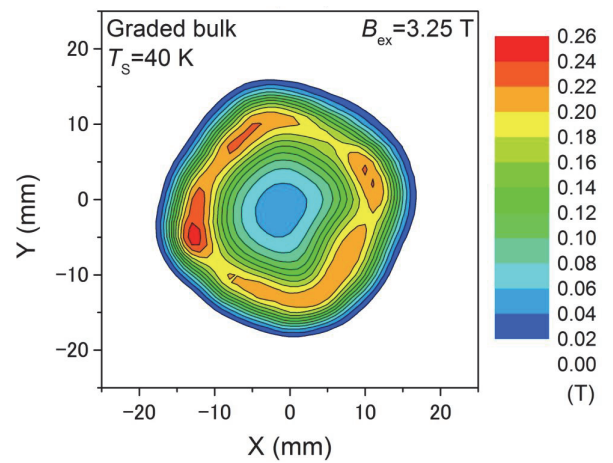

Fig. 11. 2D trapped field profile for the graded YBCO sample after PFM at $40 \mathrm{~K}$ for an applied pulsed magnetic field, $B_{\mathrm{ex}}=3.25 \mathrm{~T}$, measured at $1 \mathrm{~mm}$ above the top surface.

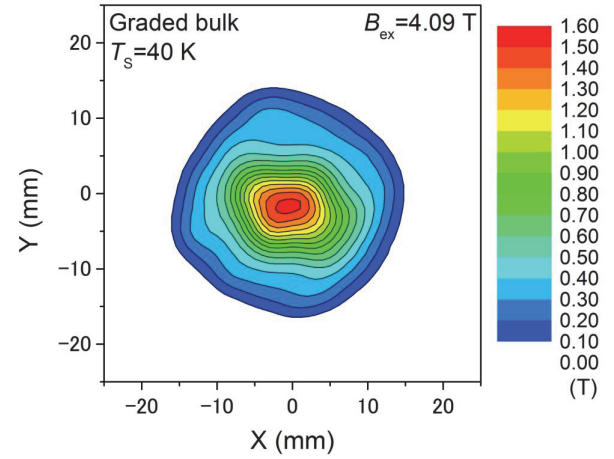

Fig. 12. 2D trapped field profile for the graded YBCO sample after PFM at $40 \mathrm{~K}$ for an applied pulsed magnetic field, $B_{\mathrm{ex}}=4.09 \mathrm{~T}$, measured at $1 \mathrm{~mm}$ above the top surface.

\section{CONCLUSION}

A new fabrication technique for melt-processed $\mathrm{Y}-\mathrm{Ba}-\mathrm{Cu}-\mathrm{O}$ (YBCO) using graded precursor powders has recently been developed by our research group, which results in a more uniform distribution of Y-211 particles, in order to further improve the superconducting properties and trapped field capability of such materials. In this paper, we examine the trapped field performance of a graded YBCO sample, fabricated using a buffer-aided, top-seeded melt-growth (TSMG) process, and compare the results to those of standard YBCO samples, using field cooling (FC) magnetization and pulsed field magnetization (PFM) techniques.

The FC magnetization results show that large, single grain bulk YBCO can be fabricated using this graded technique with trapped field capabilities equal to or better than standard samples. The PFM results show a more uniform flux penetration for the graded sample, suggesting a more homogeneous $J_{\mathrm{c}}$ distribution has been achieved, which is extremely important in order to achieve enhanced, homogeneous trapped magnetic fields from bulk superconductors. This resulted in a lower full activation field and a higher trapped field.

\section{REFERENCES}

[1] M. Tomita, and M. Murakami, "High-temperature superconductor bulk magnets that can trap magnetic fields of over 17 tesla at $29 \mathrm{~K}$," Nature, vol. 421, pp. 517-520, Jan. 2003.

[2] J. H. Durrell et al., "A trapped field of $17.6 \mathrm{~T}$ in melt-processed, bulk Gd-Ba-Cu-O reinforced with shrink-fit steel," Supercond. Sci. Technol., vol. 27, no. 8, Aug. 2014, Art. ID. 082001 .

[3] S. Nariki, N. Sakai, and M. Murakami, "Melt-processed Gd-Ba-Cu-O superconductor with trapped field of $3 \mathrm{~T}$ at $77 \mathrm{~K}$," Supercond. Sci. Technol., vol. 18, no. 2, pp. S126-S130, Feb. 2005.

[4] D. Zhou et al., "An overview of rotating machine systems with high temperature bulk superconductors," Supercond. Sci. Technol., vol. 25, no. 10 , Oct. 2012 , Art. ID. 103001.

[5] J. R. Hull and M. Murakami, "Applications of bulk high-temperature superconductors," Proc. IEEE, vol. 92, no. 10, pp. 1705-1718, Oct. 2004.

[6] M. D. Ainslie et al., "Numerical analysis and finite element modelling of an HTS synchronous motor," Physica C, Supercond., vol. 470, no. 20, pp. 1752-1755, Nov. 2010.

[7] M. D. Ainslie et al., "Design and market considerations for axial flux superconducting electric machine design," J. Phys.: Conf. Ser., vol. 507, no. 3, 2014, Art. ID. 032002.

[8] K. Yokoyama et al., "Solid-liquid magnetic separation using bulk superconducting magnets," IEEE Trans. Appl. Supercond., vol. 13, no. 2, pp. 1592-5, Jun. 2003.

[9] J. Zou et al., "Numerical simulation and analysis of single grain YBCO processed from graded precursor powders," Supercond. Sci. Technol., vol. 28, no. 3, Mar. 2015, Art. ID. 035016.

[10] W. Zhai et al., "The processing and properties of single grain $\mathrm{Y}-\mathrm{Ba}-\mathrm{Cu}-$ O fabricated from graded precursor powders," Supercond. Sci. Technol., vol. 26, no. 12, Dec. 2013, Art. ID. 125021.

[11] W. Zhai et al., "Processing and Properties of Bulk Y-Ba-Cu-O Superconductors Fabricated by Top Seeded Melt Growth from Precursor Pellets Containing a Graded $\mathrm{CeO}_{2}$ Composition," Cryst. Growth Des., vol. 15, no. 2, pp. 907-914, Jan. 2015.

[12] N. D. Kumar et al., "Buffer Pellets for High-Yield, Top-Seeded Melt Growth of Large Grain Y-Ba-Cu-O Superconductors," Cryst. Growth Des., vol. 15, no. 3, pp. 1472-1480, Feb. 2015.

[13] Y-H. Shi et al., "The use of buffer pellets to pseudo hot seed (RE)-Ba$\mathrm{Cu}-\mathrm{O}-(\mathrm{Ag})$ single grain bulk superconductors," Supercond. Sci. Technol., vol. 29, no. 1, Art. ID. 015010.

[14] J. Zou et al., "Numerical modelling and comparison of $\mathrm{MgB}_{2}$ bulks fabricated by HIP and infiltration growth," Supercond. Sci. Technol., vol. 28, no. 7, Jul. 2015, Art. ID. 075009.

[15] M. D. Ainslie et al., "Pulsed field magnetization of $0^{\circ}-0^{\circ}$ and $45^{\circ}-45^{\circ}$ bridge-seeded Y-Ba-Cu-O bulk superconductors," Supercond. Sci. Technol., vol. 28, no. 12, Dec. 2015, Art. ID. 125002.

[16] M. D. Ainslie et al., "Modelling and comparison of trapped fields in (RE)BCO bulk superconductors for activation using pulsed field magnetization," Supercond. Sci. Technol., vol. 27, no. 6, Jun. 2014, Art. ID. 065008 .

[17] Y. Shi, N. Hari Babu, and D. A. Cardwell, "Development of a generic seed crystal for the fabrication of large grain (RE)-Ba-Cu-O bulk superconductors," Supercond. Sci. Technol., vol. 18, no. 4, Apr. 2005, L13-L16.

[18] M. D. Ainslie and H. Fujishiro, "Modelling of bulk superconductor magnetization," Supercond. Sci. Technol., vol. 28, no. 5, May 2015, Art. ID. 053002 . 